\title{
Bullous Sweet's syndrome in a patient with ulcerative colitis: a rare case report
}

\author{
Melania Giannoni ${ }^{1}$, Giulio Rizzetto ${ }^{1}$, Claudia Sapigni ${ }^{1}$, Matteo Paolinelli ${ }^{1}$, Corrado Tagliati ${ }^{1}$, Federico Diotallevi ${ }^{1 凶}$, Anna \\ Campanati ${ }^{1}$, Alessandra Mandolesi ${ }^{2}$, Laura Pepi ${ }^{2}$, Annamaria Offidani ${ }^{1}$ \\ ${ }^{1}$ Department of Clinical and Molecular Sciences, Dermatology Clinic, Polytechnic Marche University, Ancona, Italy. ${ }^{2}$ Department of Experimental and \\ Clinical Medicine, Anatomopathological Institute, Polytechnic Marche University, Ancona, Italy.
}

\begin{abstract}
Bullous Sweet's syndrome is an uncommon clinical presentation of classical Sweet's syndrome, often associated with various kinds of tumors, infections, and active inflammatory bowel diseases (IBDs), namely Crohn's disease and ulcerative colitis. Only a few cases of bullous Sweet's syndrome associated with ulcerative colitis are described in the literature. We report a case of a 62-year-old female patient with acute exacerbation of ulcerative colitis associated with infiltrating purple-erythematous skin plaques, which were partly vesicular, and oral ulcerative stomatitis. Biopsy was consistent with bullous Sweet's syndrome. Treatment with betamethasone sodium phosphate, starting at $5.5 \mathrm{mg}$, followed by gradual dose tapering for 12 weeks, resulted in improvement of the ulcerative colitis and disappearance of the cutaneous lesions. Bullous Sweet's syndrome most commonly occurs in the setting of hematologic malignancies, suggesting that physicians should perform long-term screening for early diagnosis of hematological and solid malignancies.
\end{abstract}

Keywords: Bullous Sweet's syndrome, Crohn's disease, ulcerative colitis

Received: 15 March 2020 | Returned for modification: 16 April 2020 | Accepted: 20 April 2020

\section{Introduction}

Sweet's syndrome (also known as acute febrile neutrophilic dermatosis) is a rare dermatosis characterized by a combination of physical features, clinical symptoms, and pathological specimens, including tender erythematous skin lesions (papules, nodules, and plaques) located on the arms, upper body, head and neck; high-grade fever; neutrophilia; and an extensive infiltration of predominantly mature neutrophils that are typically located in the upper dermis (1).

Based on pathophysiology, three subgroups are identified: idiopathic, malignancy-associated, and drug-induced (1).

Bullous Sweet's syndrome is a rare clinical variant often associated with various kinds of tumors (hematological and solid tumors, including breast, ovarian, testicular, prostatic, rectal, and colon adenocarcinoma), infections, and active inflammatory bowel diseases (IBDs; Crohn's disease and ulcerative colitis) (2-4).

\section{Case report}

We report the case of a 62-year-old female with active ulcerative colitis that visited our dermatologic unit in September 2019 for a sudden onset of fever, gastrointestinal symptoms, and painful skin lesions 10 days after the interruption of oral corticosteroids for unreported reasons.

On physical examination, infiltrating purple-erythematous skin plaques, which were partly vesicular, were evident on her lower and upper limbs (Fig. 1a, 1b). The oral mucosa was involved contemporarily with ulcerative stomatitis (Fig. 2). Both erythrocyte sedimentation rate (ESR) and C-reactive protein (CRP) were elevated (6o mm/h and $15 \mathrm{mg} / \mathrm{l}$, respectively), as well as the neutrophil blood count $\left(10,000 \mathrm{~mm}^{3}\right)$.

The patient did not report recent intake of new drugs or infections in the previous few weeks. Blood, urine and fecal cultures, throat swab for Streptococcus pyogenes, and viral serology for herpesvirus 1 and 2, cytomegalovirus, and Epstein-Barr virus were negative for recent infections. Histologic examination of biopsy specimens obtained from perilesional skin of the lower limbs showed marked edema of papillary dermis associated with subepidermal vesiculation (Fig. 3). Marked inflammatory infiltrate, predominantly neutrophilic granulocytes, is found in the papillary and reticular dermis, mainly perivascular and around the ducts of sweat glands (Fig. 3). Intraepidermal neutrophilic exocytosis was minimally present. Direct immunofluorescence of frozen perilesional tissue did not demonstrate IgA, IgG, IgM, $\mathrm{C}_{3}$, or fibrinogen deposits. Based on clinical and histological features, the diagnosis of Sweet's syndrome was made.

We treated our patient with intravenous daily administration of betamethasone sodium phosphate, starting from $5.5 \mathrm{mg}$, followed by gradual dose tapering for 12 weeks consecutively. Systemic administration of high-potency corticosteroids resulted in rapid disappearance of both fever and gastrointestinal symptoms within 2 days, followed by gradual clinical resolution of skin eruption and great improvement of oral involvement.

Currently, 4 months after acute illness, both Sweet's syndrome and ulcerative colitis are in complete clinical remission (Fig. 4), as confirmed by post-therapy colonoscopy (MAYO o-1). The patient is still continuing chronic anti-inflammatory treatment with oral mesalazine at $2.4 \mathrm{~g}$ once a day. 

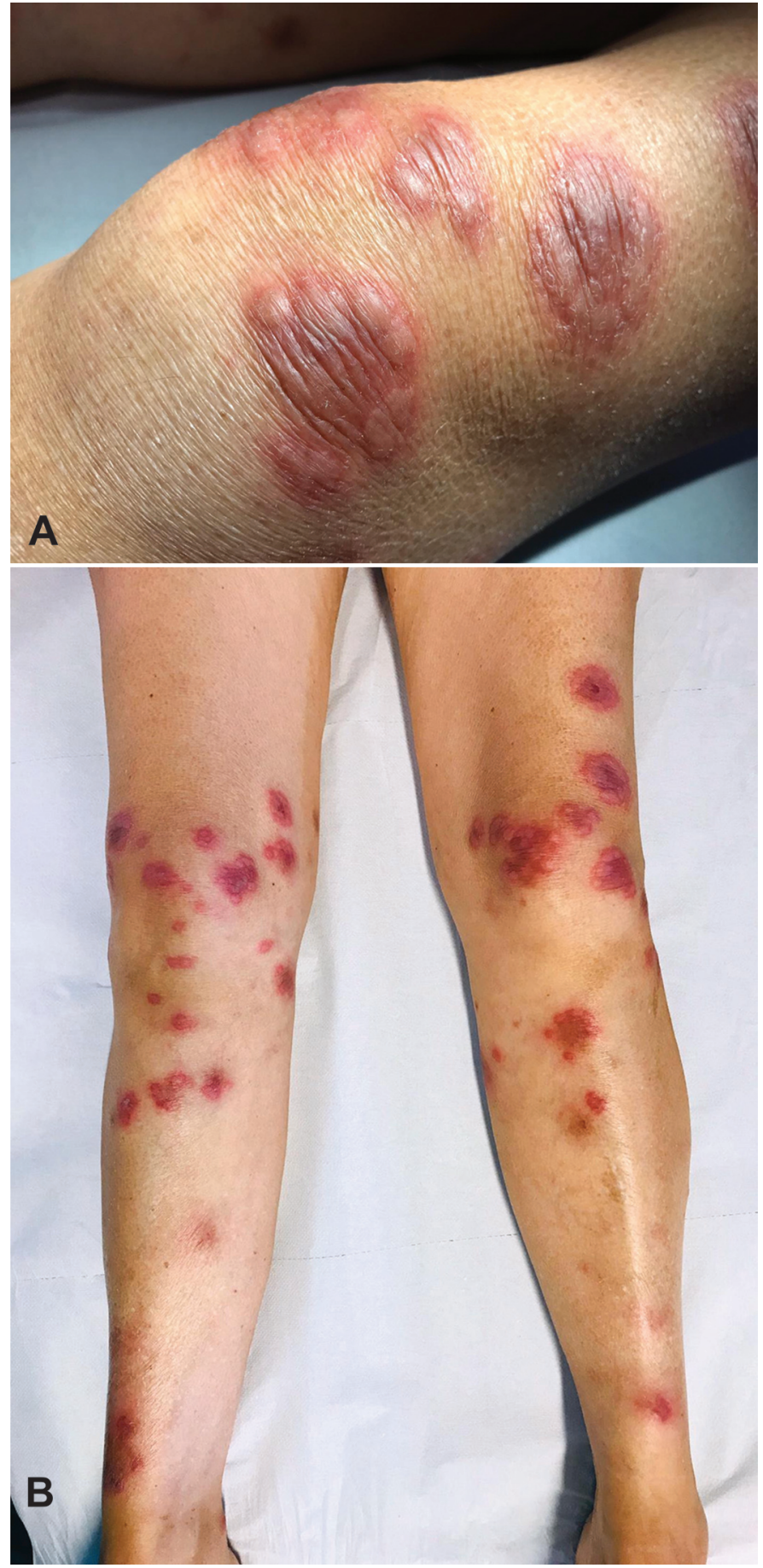

Figure 1 | Purple-erythematous infiltrating skin plaques with vesicular features located on the lower extremities.

\section{Discussion}

Bullous lesions are quite an uncommon clinical variant of Sweet's syndrome. Moreover, although IBDs are frequently associated with the classical variant of Sweet's syndrome, only a few cases of bullous Sweet's syndrome associated with IBD have been already described in the literature (3-9).

In our case, anatomopathological examination of skin lesions supported the diagnosis of this rare variant of Sweet's syndrome, excluding the presence of pseudovesicular lesions due to edema. Sweet's syndrome occurs most frequently during periods of acute exacerbation of IBDs, but it may also precede bowel disease onset (3). Accordingly, it is reasonable not only to investigate an undiagnosed neoplasm at baseline, but also to monitor intestinal

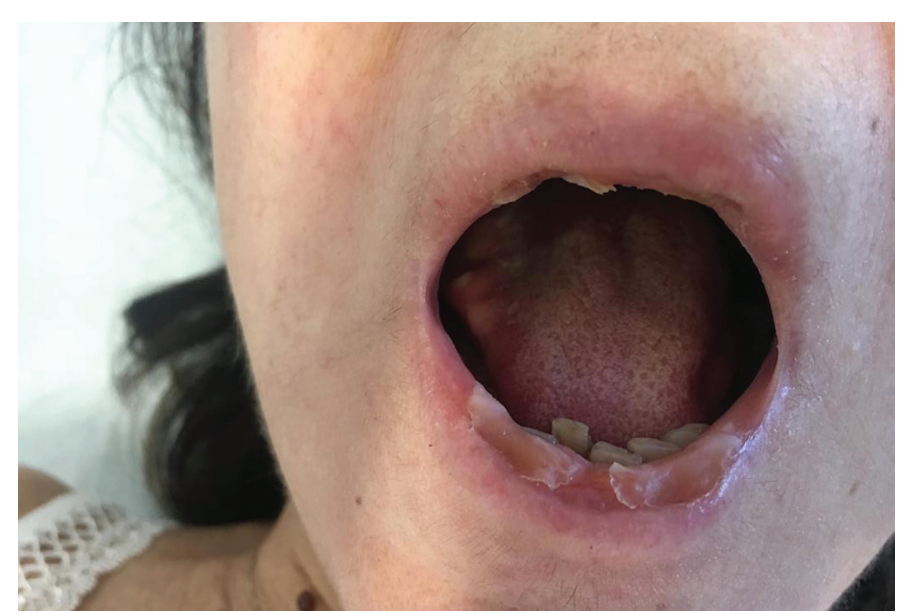

Figure 2 | Oral mucosa involvement characterized by ulcerative lesions on the lower lip.

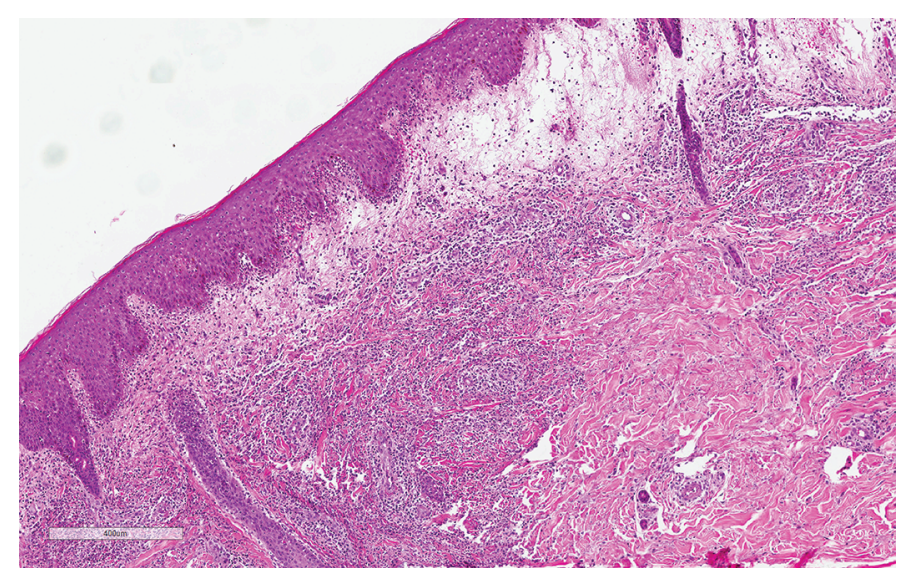

Figure 3 | Histological examination of skin specimen, H\&E (100x). Both papillary and reticular dermis showed marked inflammatory infiltrate, predominantly neutrophilic. Intraepidermal neutrophilic exocytosis was minimally present.

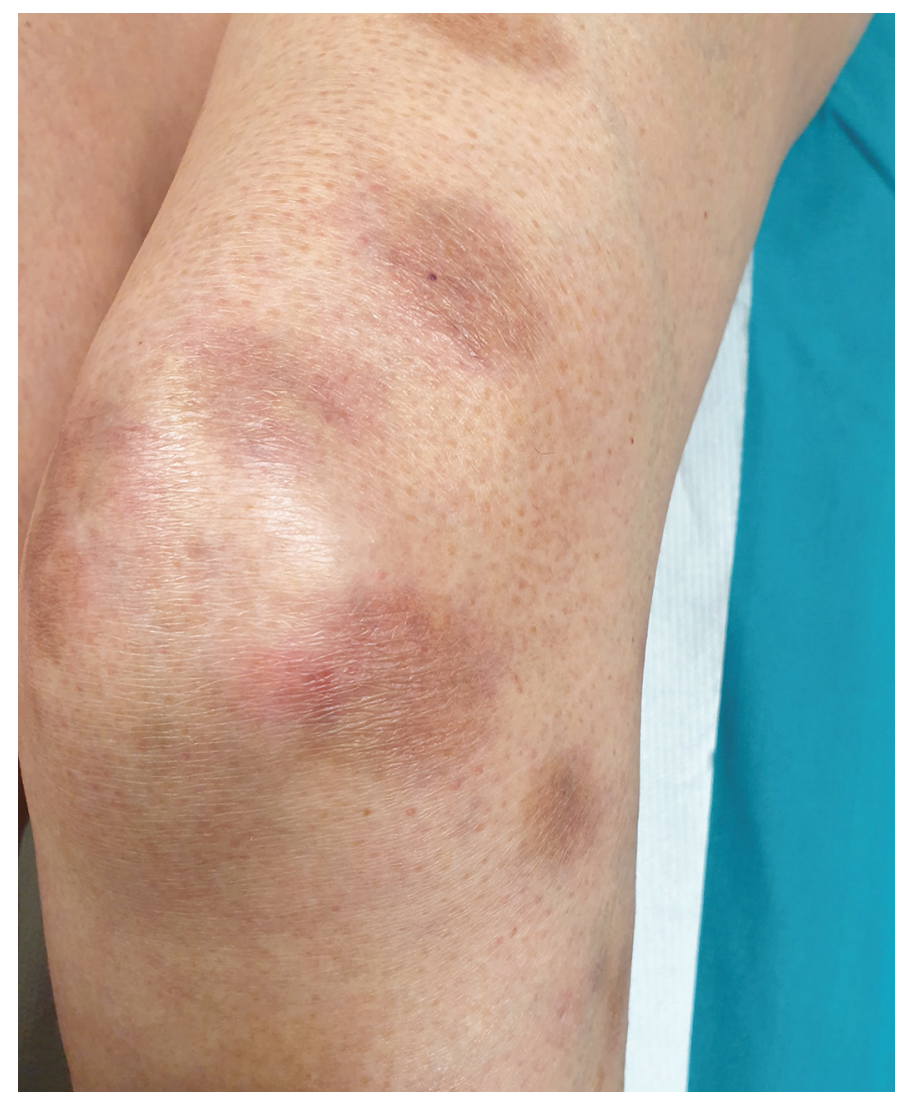

Figure 4 | Complete clinical remission of skin lesions after systemic administration of high-potency corticosteroids. 
status in the years following the appearance of this skin disease, particularly in patients at high risk for intestinal bowel disease. As Raza et al. (10) recently described, bullous Sweet's syndrome most commonly occurs in the setting of hematologic malignan- cies, which can be a premonitory paraneoplastic syndrome. Currently our patient has been included in long-term screening for early diagnosis of hematological and solid malignancies.

\section{References}

1. Cohen PR. Sweet's syndrome-a comprehensive review of an acute febrile neutrophilic dermatosis. Orphanet J Rare Dis. 2007;26:34.

2. Endo Y, Tanioka M, Tanizaki H, Mori M, Kawabata H, Miyachi Y. Bullous variant of Sweet's syndrome after herpes zoster virus infection. Case Rep Dermatol. 2011; 3:259-62.

3. Nasa M, Sharma Z, Lipi L, Sud R. Sweet's syndrome in a case of ulcerative colitis-case report and review of literature. J Assoc Physicians India. 2019;67:84-5.

4. Marzano AV, Ishak RS, Saibeni S, Crosti C, Meroni PL, Cugno M. Autoinflammatory skin disorders in inflammatory bowel diseases, pyoderma gangrenosum and Sweet's syndrome: a comprehensive review and disease classification criteria. Clin Rev Allergy Immunol. 2013;45:202-10.

5. Esposito I, Fossati B, Peris K, De Simone C. A rare case of bullous Sweet's syndrome in a patient with inactive ulcerative colitis. J Eur Acad Dermatol Venereol. 2019;33:e380-1.

6. Wells MM, Stecho W, Wehrli B, Khanna N. Sweet syndrome secondary to inflammatory bowel disease. Can J Gastroenterol. 2013;27:124-5.

7. Greuter T, Navarini A, Vavricka SR. Skin manifestations of inflammatory bowel disease. Clin Rev Allergy Immunol. 2017;53:413-27.

8. Ali M, Duerksen DR. Ulcerative colitis and Sweet's syndrome: a case report and review of the literature. Can J Gastroenterol. 2008;22:296-8.

9. Raza S, Kirkland RS, Patel AA, Shortridge JR, Freter C. Insight into Sweet's syndrome and associated-malignancy: a review of the current literature. Int J Oncol. 2013;42:1516.

10. Campanati A, Offidani A. Textbook of dermatology \& sexually transmitted diseases. Padua: Piccin; 2014. Chapter 131, Mucocutaneous paraneoplastic syndromes; p. 2327-44. 